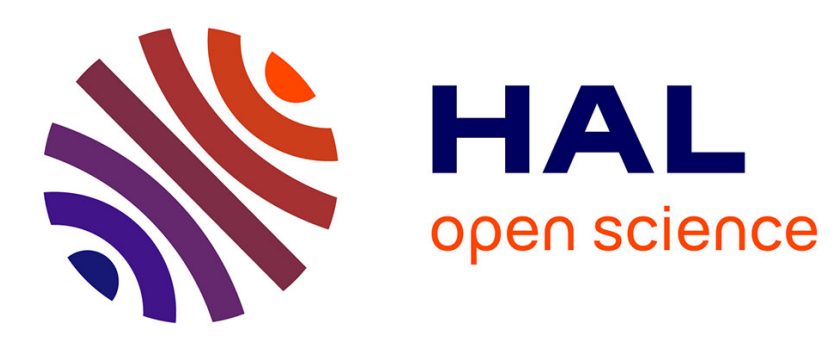

\title{
On Fairness and Locality in P2P-TV through Large-Scale Measurement Experiment
}

Thomas Silverston, Olivier Fourmaux, Kavé Salamatian, Kenjiro Cho

\section{To cite this version:}

Thomas Silverston, Olivier Fourmaux, Kavé Salamatian, Kenjiro Cho. On Fairness and Locality in P2P-TV through Large-Scale Measurement Experiment. GLOBECOM 2010 - IEEE Global Telecommunications Conference, Dec 2010, Miami, United States. pp.1-6, 10.1109/GLOCOM.2010.5683556 . hal-00527126

\section{HAL Id: hal-00527126 \\ https://hal.science/hal-00527126}

Submitted on 18 Oct 2010

HAL is a multi-disciplinary open access archive for the deposit and dissemination of scientific research documents, whether they are published or not. The documents may come from teaching and research institutions in France or abroad, or from public or private research centers.
L'archive ouverte pluridisciplinaire HAL, est destinée au dépôt et à la diffusion de documents scientifiques de niveau recherche, publiés ou non, émanant des établissements d'enseignement et de recherche français ou étrangers, des laboratoires publics ou privés. 


\title{
On Fairness and Locality in P2P-TV through Large-Scale Measurement Experiment
}

\author{
Thomas Silverston*, Olivier Fourmaux ${ }^{\dagger}$, Kavé Salamatian $^{\ddagger}$ and Kenjiro Cho ${ }^{\S}$ \\ *The University of Tokyo, JFLI/CNRS \\ †UPMC Univ Paris 6, LIP6/CNRS, UMR 7606 \\ $\ddagger$ Universite de Savoie Chambery Annecy, LISTIC PolyTech \\ $\S$ IIJ Research Lab, Tokyo, Japan
}

\begin{abstract}
In this paper, we present our P2P-TV measurement experiment performed in France and in Japan. By using multiple measurement points in different locations of the world, we are able to get a global view of the measured P2P networks and we can infer their main properties. More precisely, we focus on the level of collaboration between peers, their location and the effect of the traffic on the networks. Our results show that there is no fairness between peers and it is an important issue for the scalability of P2P-TV systems. Moreover, hundreds of Autonomous Systems are involved in the P2P-TV traffic and it points out the lack of locality-aware mechanisms for these systems. The geographic location of peers testifies the wide spread of these applications in Asia and highlights their worldwide usage.
\end{abstract}

\section{INTRODUCTION}

Peer-to-peer video live streaming applications (P2P-TV) emerged recently as a new framework to deliver live video such as television over the Internet. The quick spread of these applications surprisingly show that user oriented technologies based on collaboration between similar users without a central control entity is capable to deliver delay sensitive multimedia content. As a consequence, the Internet counts today several of these applications such as PPSTream [1], SOPCast [2], TVUPlayer [3] or TVAnts [4]. Millions of users located all over the globe watch lively hundreds of channels.

The P2P model, essentially known for its scalability, is a practical solution for broadcasting live events or TV shows to a large number of receivers without any deployment cost as it is the case with content distribution network [5] (CDN). Nowadays, P2P traffic contributes largely to the Internet traffic [6]. The same observation applies on video streaming traffic generated by platforms such as Youtube [7]. Thus, P2P-TV applications that combine these two technologies are expected to count for a large part of the Internet traffic.

However, the main problem remains in characterizing the unknown effect of P2P video streaming traffic on the Internet and on Internet Service Providers (ISPs). It was already shown that the traditional P2P traffic is a serious threat for ISPs [8]. Nevertheless P2P video streaming traffic, which consumes a lot of bandwidth resources and is very sensitive to the end-to-end delay, is a more intriguing case. Moreover, the fact that television services target a huge number of users spread worldwide further complicates traffic engineering tasks for ISPs. Therefore, it is of a great significance to better characterize the impact of $\mathrm{P} 2 \mathrm{P}-\mathrm{TV}$ traffic on the Internet and ISPs networks [9].

Numerous P2P-TV measurement experiments focused mainly on the reverse engineering of these commercial applications [10], [11], [12]. Because most of these experiments studied the traffic from a single measurement point, as it was the case with our previous work [13], the main goal of these works was to infer the underlying mechanisms or architectures used by these proprietary applications. However, these applications are used at the planet-scale and the geographic location of peers, the users' interest in content according to its location, and their Internet access environment have an impact on the behavior of users and the properties of collected traffic.

In this paper, we present a large-scale measurement experiment of P2P-TV systems. We collect the traffic from multiple measurement points located in France and in Japan (Section 2). Through this measurement experiment, we study the overall P2P-TV networks and extract new characteristics relevant for ISPs and application designers. We study the global organization of the peers and the amount of traffic they exchange between them. It leads to uncover the lack of fairness between peers in exchanging data (Section 3). We also study the distance among them and discuss the number of ASes involved in the traffic and the impact on ISPs. It points out the lack of locality-aware mechanisms that make P2P-TV traffic a dilemma for ISPs since hundreds of ASes are involved in the traffic (Section 4). We also provide a comprehensive study on the geographic location of users and the impact of the content on the population of P2P-TV users (Section 5).

\section{LARGE-SCALE EXPERIMENTAL TESTBED}

For our measurement experiments, we passively collected the traffic from multiple points located in France and in Japan. We focus on the most popular P2P-TV applications, namely PPSTream, TVUPlayer, SOPCast and TVAnts. We selected these applications according to our feedbacks and those from the online community [14]. During our experiments, we measured live soccer games because such events exhibit a real interest to be watched lively. There is also a large community of P2P-TV users for this purpose.

Our measurement testbed is described in Fig 1 and is composed of two distinct parts situated in France and in Japan. In each part, we collect packets by using PCs equipped 
TABLE I

P2P-TV TRAFFiC TRACES. All the traces haVe the SAME DURATION: 2H45 (165Min).

\begin{tabular}{|c|c|c|c|c|c||c|c|c|c|c|c|}
\hline PPSTream & $\begin{array}{c}\text { Size } \\
(\mathrm{GB})\end{array}$ & \#IP & $\begin{array}{c}\text { Simi- } \\
\text { larity }(\%)\end{array}$ & $\begin{array}{c}\text { Up. } \\
(\% \text { Size })\end{array}$ & $\begin{array}{c}\text { Down. } \\
(\% \text { Size) }\end{array}$ & TVUPlayer & $\begin{array}{c}\text { Size } \\
(\mathrm{GB})\end{array}$ & \#IP & $\begin{array}{c}\text { Simi- } \\
\text { larity }(\%)\end{array}$ & $\begin{array}{c}\text { Up. } \\
(\% \text { Size) }\end{array}$ & $\begin{array}{c}\text { Down. } \\
(\% \text { Size })\end{array}$ \\
\hline France A & 3.1 & 2,625 & 68 & 82 & 18 & France A & 1.7 & 2,122 & 46 & 66 & 34 \\
\hline France B & 3.1 & 3,317 & 57 & 82 & 18 & France B & 1.6 & 1,262 & 66 & 68 & 32 \\
\hline France C & 3.1 & 3,224 & 59 & 82 & 18 & France C & 1.6 & 1,093 & 70 & 67 & 33 \\
\hline Japan X & 3.0 & 3,421 & 54 & 82 & 18 & Japan X & 1.8 & 1,111 & 89 & 67 & 33 \\
\hline Japan Y & 3.1 & 2,544 & 67 & 82 & 18 & Japan Y & 2.2 & 1,034 & 90 & 71 & 29 \\
\hline TVAnts & Size & \#IP & $\begin{array}{c}\text { Simi- } \\
\text { larity }(\%)\end{array}$ & $\begin{array}{c}\text { Up. } \\
(\%)\end{array}$ & $\begin{array}{c}\text { Down. } \\
(\%)\end{array}$ & Japan Z & 3.1 & 1,064 & 92 & 78 & 22 \\
\hline France A & 2.7 & 1,854 & 97 & 79 & 21 & SoPCast & $\begin{array}{c}\text { Size } \\
(\mathrm{GB})\end{array}$ & \#IP & $\begin{array}{c}\text { Simi- } \\
\text { larity }(\%)\end{array}$ & $\begin{array}{c}\text { Up. } \\
(\% \text { Size })\end{array}$ & $\begin{array}{c}\text { Down. } \\
(\% \text { Size })\end{array}$ \\
\hline France B & 2.0 & 1,864 & 97 & 72 & 28 & France A & 1.0 & 3,755 & 79 & 43 & 57 \\
\hline France C & 2.6 & 1,768 & 97 & 78 & 22 & France B & 1.2 & 4,268 & 73 & 50 & 50 \\
\hline France D & 2.7 & 1,887 & 97 & 79 & 21 & France C & 1.2 & 3,920 & 76 & 53 & 47 \\
\hline Japan X & 2.4 & 1,855 & 97 & 77 & 23 & France D & 1.0 & 3,925 & 80 & 41 & 59 \\
\hline Japan Y & 2.3 & 1,862 & 97 & 75 & 25 & Japan X & 3.2 & 4,269 & 81 & 78 & 22 \\
\hline Japan Z & 2.5 & 1,877 & 97 & 78 & 22 & Japan Y & 3.0 & 4,048 & 83 & 77 & 23 \\
\hline
\end{tabular}

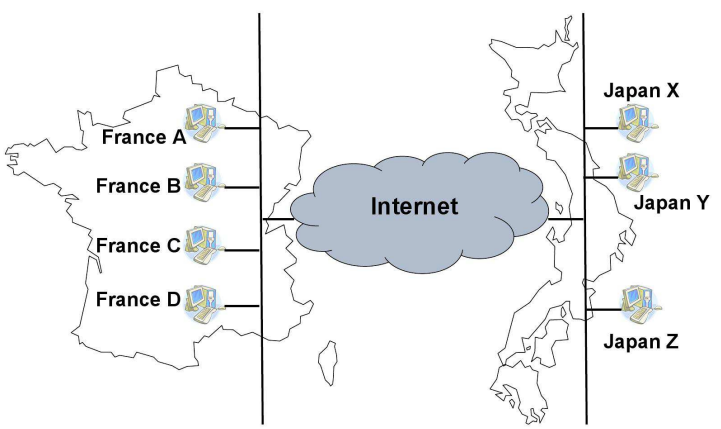

Fig. 1. Large-scale measurement experiments testbed. Each PC is directly connected to the Internet within UPMC or the University of Tokyo network.

with $1.8 \mathrm{GHz} \mathrm{CPU}$, common graphic card capabilities, and Windows XP. For each of the four measured applications, we performed an experiment involving different number of PCs according to their availability (5 to $7 \mathrm{PCs}$ at the same time). From three to four PCs were situated in the UPMC campus network in France and were directly connected to the Internet through a 100Mbps Ethernet link. We used two to three PCs in the campus network of the University of Tokyo in Japan, also directly connected to the Internet (Ethernet 100Mbps). During an experiment, all the PCs were running the same P2P-TV application as well as Windump to collect the packets. All the video bitrate were $400 \mathrm{Kbps}$.

The table I summarizes the collected traces ${ }^{1}$. All the traces have the same duration of $2 \mathrm{~h} 45 \mathrm{~min}$. This duration is slightly larger than a soccer game (105 minutes) because we wanted to capture the effect that happens at the beginning or the end of the games. For clarity reasons, we refer to the PCs situated in France as France $A-D$ and those in Japan as Japan $X$ $Z$. PPStream was measured with five PCs (nodes) during the UEFA Champion's league between Liverpool and Toulouse (08/15/2007). Six nodes measured TVUPlayer during a quali-

\footnotetext{
${ }^{1}$ The traces will be publicly available on our sharing platform: http://content.lip6.fr/traces/
}

fying game for the Olympic soccer tournament between Japan and Vietnam (08/22/2007). For SOPCast, we used six nodes during a similar event but with China and Japan (08/03/2007). TVAnts was measured with all the seven nodes for another qualifying game between China and Vietnam (08/23/2007).

\section{A. Data Set Observations}

We present on table I some statistical properties of the traces such as their size, or number of IPs (i.e remote peers) encountered in each trace. We also compute a similarity measure (described hereafter) and the ratio of upload and download traffic.

The upload ratio is more important for large traces than for the smaller one and it testifies to a more important upload activity. Indeed, a peer aims to download the video only once but can upload it several times to remote peers. Regarding the volume of download, it may range from $512 \mathrm{MB}$ for France B to $682 \mathrm{MB}$ for Japan $\mathrm{Z}$ with TVUPlayer, while the traces have the same duration $(2 \mathrm{~h} 45)$. This additional amount of downloaded traffic comes from the signaling traffic generated by the remote peers. This observation is an important issue in several scenarios. An altruistic peer serving video will receive in return a large amount of signaling traffic from those remote peers orchestrating their download. It will waste its bandwidth resource to download the video and could directly affect the video quality if its access link becomes saturated.

In order to understand if the number of measurement points (5 to 7) was sufficient to obtain a global view of the P2P network, we computed a "similarity measure". The similarity measure is defined as the ratio of IPs from a trace that are also present in the other traces of the same application. For instance, France B with PPSTream accounts 3,317 IPs where $57 \%$ of those are also present in the other PPSTream traces. For PPStream -5 nodes- in average $61 \%$ of IPs are also present in its other traces. The average similarity for TVUPlayer is $75 \%$ and $79 \%$ for SOPCast (6 nodes) and $97 \%$ for TVAnts (7 nodes). We observed that the more measurement points we had, the more our nodes communicated with similar sets 


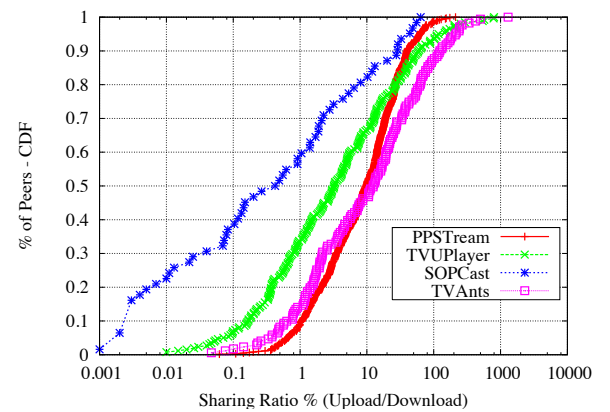

Fig. 2. Traffic sharing ratio (Upload/Download)

of peers. This validate that the observed high similarity ratio indicates that our nodes encountered the entire population of peers in the network, leading to a global and precise view of the P2P network.

Our experimentation showed that only 7 measurements points allowed us to attain a similarity measure of $97 \%$. This lead us to believe that this order of magnitude of measurement points is adequate to measure entirely a P2P network. This is an important observation as it shows that there is no need to measure the network with a very large number of peers (hundreds, thousands) in order to have a precise view of the network. In our experiments the cases with 5 to 6 nodes are not as precise as with 7 nodes. However they give a much more precise and global view of the P2P networks when compared with previous studies with only a single measurement point [13].

\section{iII. Collaboration Between Peers}

In P2P systems, peers are responsible to duplicate the content to others. The overall P2P networks rely on the effective collaboration of users. In order to evaluate the level of collaboration between peers, we compute for each peer the "sharing ratio". The sharing ratio of a peer is the amount of traffic it uploads divided by the amount of traffic it downloads.

For all applications, figure 2 shows the cumulative distribution function (CDF) of the relationship between the sharing ratio (upload/download) between a peer which we control, and all the peers with which it exchanges data. We show a single trace for each application because it is representative for the other traces. When the ratio is above 1, our peer is altruistic towards another peer (i.e., our peer sends more data than it receives); when equal to 1 , the exchange is fair; when below one, our peer is taking advantage of the altruism of others.

For PPStream and TVAnts, $10 \%$ of peers are altruistic with our peer and transmitted more data than they received. The $90 \%$ remaining are beneficiaries of our altruism. This behavior is the same for TVUPlayer at distinct rate: $30 \%$ of peers are altruistic and the remaining $70 \%$ benefits from our altruism. SOPCast shows the opposite behavior: the majority of the remote peers transmitted more data than they received (60\%).

Overall, the traffic exchanges for all the applications are never fair to our controlled peers, with the large majority being mainly consumers (ratio $>1)$ than producers $($ ratio $<1$ ). SOPCast presents also an unfair sharing ratio since a large

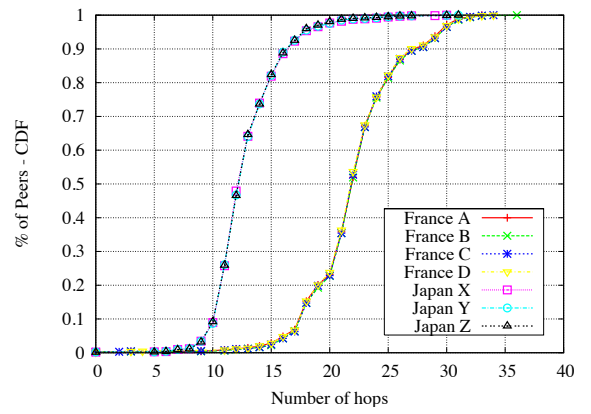

Fig. 3. Distance in number of hops (Ex: TVAnts)

majority of peers are altruistic with our peers. These results show that fairness is not achieved in P2P-TV systems. Since each peer is in charge to forward the traffic to other peers, each peer should forward the same amount of data it received. If it is not the case, the peers are not helping the other peers by sharing their resources in the network and this is a major issue for the scalability of these P2P systems.

\section{Locality of PeERS}

In this section, we study the distance from the remote peers to ours to fetch the video. We study this distance at the IP level and at the AS level.

\section{A. Distance in IP hops}

In order to infer the distance between peers, we investigate the IP datagram of the packets. The IP header has a TTL field, which is decreased at each hop by the Internet routers. This piece of information reveals the distance in number of hops from the source to the destination. Given that most of the measured applications are built for MS-Windows, which sets the default value for the TTL field to 128, the chances are that, if a packet arrives with a TTL of 120, the packet was originally sent with a TTL of 128 [15]. The distance in number of hops between the source and the receiver of the IP datagram is therefore 8 hops.

The figure 3 shows the $\mathrm{CDF}$ of the distance in number of hops between the source and the destination. All the applications show the same results and we present only those for TVAnts because of space limitations. For our French nodes, $75 \%$ of the remote peers are situated from 20 to 30 hops. For the Japanese nodes, $80 \%$ of the remote peers are situated from 10 to 16 hops. Clearly, our French peers download the video at further distance than the Japanese peers.

Several facts can explain this trend. First, P2P-TV applications have been released by Chinese companies and are already very popular in Asia. Most of the peers and source servers are probably situated in Asia. The use of P2P-TV in Europe is still limited and even PPSTream, which was broadcasting a program of interest for European users (UEFA Champions' league), has the same behavior as the other applications. Consequently, if the large majority of peers are situated in Asia, the French nodes must fetch the content from peers at further distance compared with the Japanese nodes. Second, these applications do not use any locality-aware mechanisms to select the provider peers. Otherwise, the French nodes would 


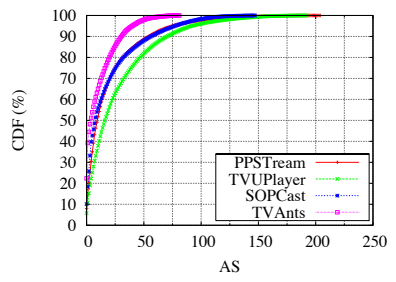

Fig. 4. Upload traffic by ASes

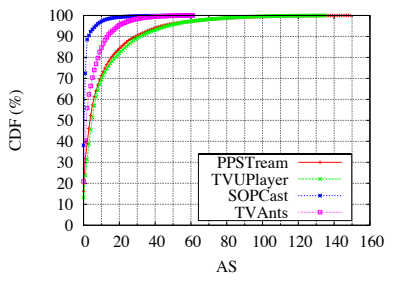

Fig. 5. Download traffic by ASes

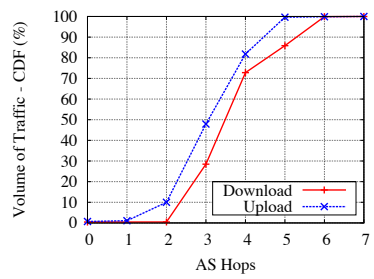

Fig. 6. AS hops (Ex: SOPCast) have downloaded the video at a closer distance. A possible scenario could be that only a single French node downloads the video from a far distance and then forwards the content to the other French nodes situated in the same network.

The long distance for the peers to get the video is a critical issue since it may increase the end-to-end delay to receive the video packets and affect the quality of the video. This also point out the fact that traffic is exchanged through different ISPs and crosses inter-ISPs links to reach distant destinations.

\section{B. Autonomous Systems}

We investigate the amount of traffic exchanged between different autonomous systems (ASes). Even though the network of ISPs can be divided into several ASes, the number of ASes involved in the exchanges provides insights on the traffic between ISPs. To this end, we mapped each IP address to its parent AS by using the whois [16] service provided by Cymru [17] and we aggregate the traffic by ASes in upload (Fig.4) and download (Fig. 5). We present one trace by application because the other traces from the same application show similar results.

Hundreds of ASes are implied in the traffic exchanges, which indicates a large dispersion of peers. This is even more pronounced in upload than download. PPStream and TVUPlayer involve approximately 200 ASes in upload, 150 for SOPCast. For PPStream and SOPCast, $90 \%$ of the upload traffic goes towards 60 different ASes. The traffic of TVUPlayer is more spread: $90 \%$ of its upload traffic goes to 75 ASes. For TVAnts, only 30 ASes count for $90 \%$ of its upload traffic: half as many ASes as the other applications. For all the applications, the downloaded traffic comes from a much smaller set of ASes than the upload traffic. For PPSTream and TVUPlayer, $90 \%$ of the downloaded traffic comes from 30 ASes, 10 ASes for TVAnts and only 3 ASes for SOPCast.

In order to obtain the number of AS hops between our controlled nodes and the other peers on the Internet we performed, during the experiments, a traceroute from our controlled peers towards each destination. It allows discovering the route packets take to reach the host destinations. From the traceroute results, we obtained the corresponding AS of each network interface of a route by using the whois service. By aggregating the similar consecutive AS of a route, we get the AS path between our nodes and each destination. The traceroutes have been performed only from our nodes situated in France since this implicates peers that download the video far from them in term of network hops.

We present for a French trace of SOPCast (Fig. 6) the CDF of the traffic according to the number of AS hops. French traces of all applications show similar behavior. We observe that $50 \%$ of the upload traffic is within a radius of $3 \mathrm{AS}$ hops whereas only $30 \%$ of the download traffic come from this distance. $15 \%$ of the traffic is downloaded at a distance ranging from 5 to 7 AS hops while upload traffic never reach destinations beyond 5 AS hops.

We noticed previously that the nodes in France download the video at farther distance than the Japanese one. Moreover, a large number of ASes are involved in the exchanges. Consequently, for the French nodes, the download traffic has transited into much more ASes compared with the upload traffic. These results highlight that P2P-TV systems do not consider the locality of peers when exchanging traffic. It is a critical issue for ISPs and it imposes significant traffic engineering challenges. For ISPs, it is important to keep the P2P traffic local. Otherwise, It may overload the links between ISPs, which are already considered as the network bottlenecks [18]. These inter-ISPs links are also very costly and ISPs aim to limit the use of these links to their customers. Clearly, service providers need real incentives to use such links to convey traffic to peers from other ISPs.

\section{Geographic Location of Peers}

In this section, we study the geographic location of peers and the volume of traffic exchanged by countries. We mapped a peer's IP address to its origin country by querying the free MaxMind GeoIP database [19]. For each trace, we separate the upload and download and present the volume of traffic and the population by countries. Each trace is therefore represented by 4 stacked histograms as shown in figure 7 . The countries counting for less than $1 \%$ of the traffic are gathered under the label "other" on the legend. For a given trace, there may be differences between the population in upload or in download since peers may be active only one side.

As it was expected, the broadcasted event has an effect on the geographic location of peers present in the traces. We observe an important number of peers from United Kingdom with PPSTream because a soccer games with the Liverpool team was broadcasted. There are also many peers from China with SOPCast and TVAnts, or from Japan with SOPCast and TVUPlayer.

\section{A. Population vs. Volume of Traffic}

There is not necessarily a relationship between the population of peers and the volume of traffic exchanged by country. In the PPSTream traces (figure 7(a)), China represents $30 \%$ of the peers that generates from $10 \%$ to $20 \%$ of traffic in upload. Chinese peers in France B (SOPCast) represents $40 \%$ of the 


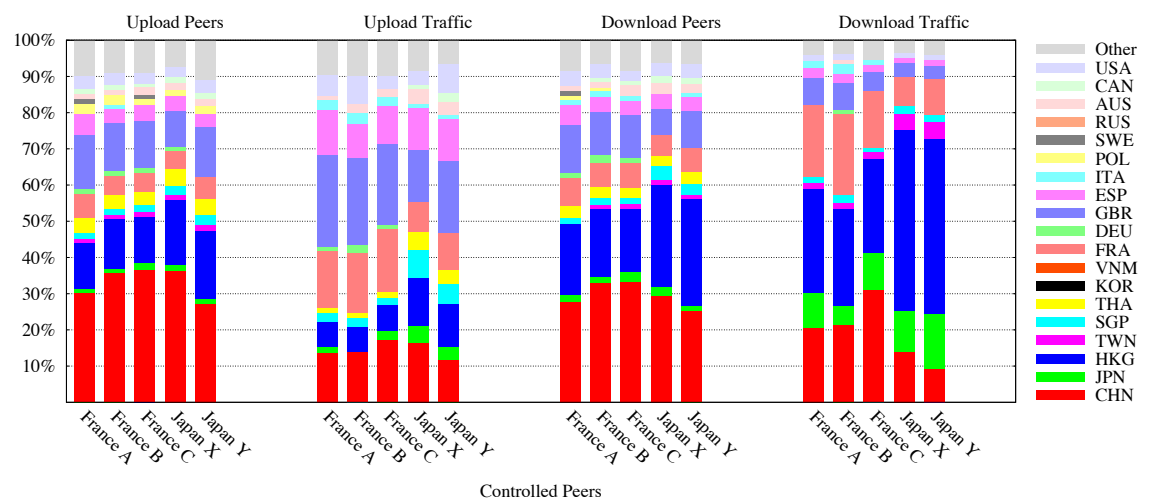

(a) PPSTream

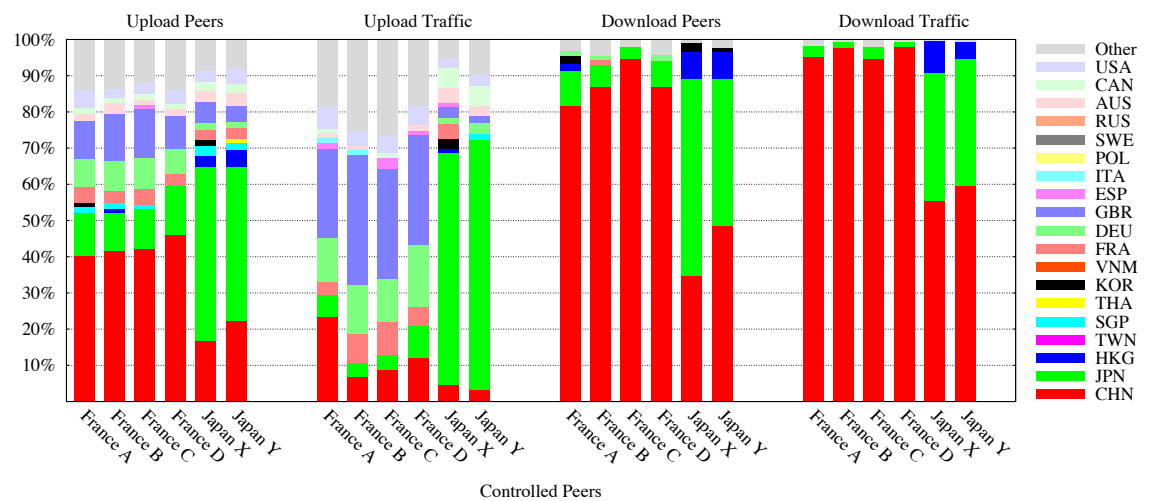

(b) SOPCast

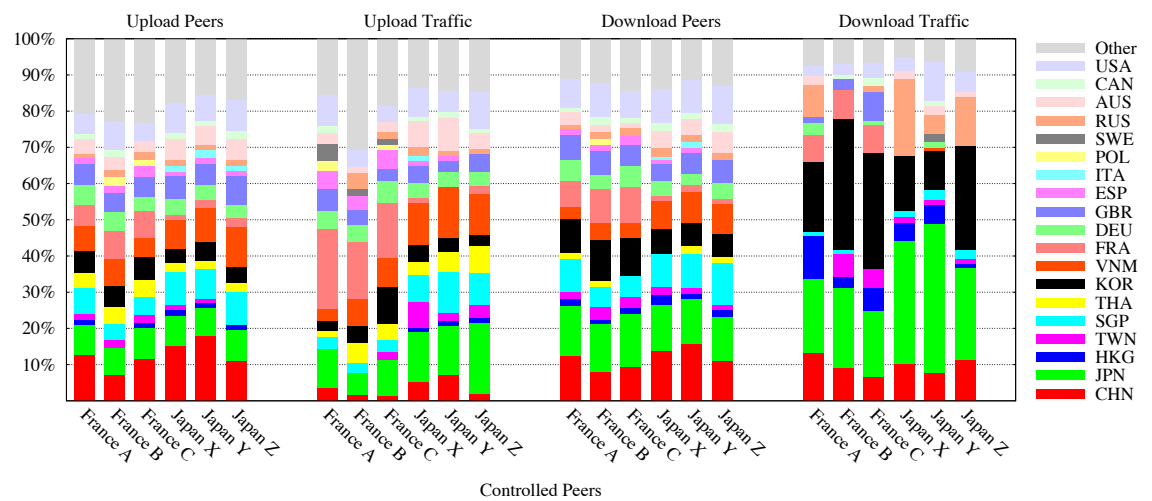

(c) TVUPlayer

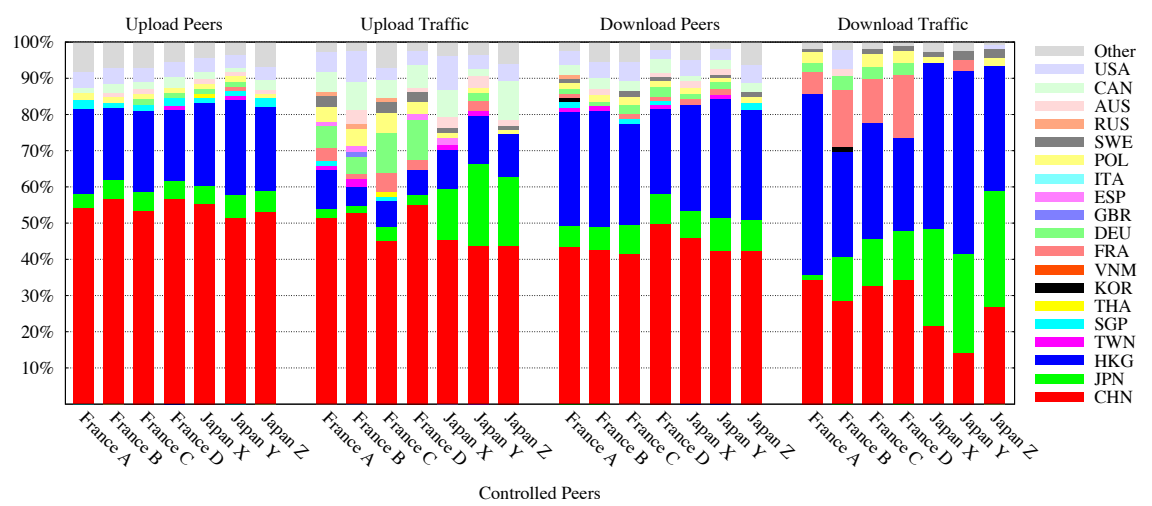

(d) TVAnts

Fig. 7. Geographic location of traffic and peers 
population in upload (figure 7(b)) but only $8 \%$ of the volume of traffic. This statement is also verified in TVUPlayer or TVAnts. Among all the measured applications, SOPCast shows a particular behavior in download where only a few countries are involved in the exchanges. In the download side, our nodes fetch the content almost entirely from Asia. Concerning upload, the traffic is spread into much more countries: towards Europe for our French nodes and Asia for the Japanese nodes.

\section{B. Impact on the collaboration of Peers}

From the previous observation, we noticed that at the country-level, our nodes do not trade equitably the data. Intuitively, this result can be extended to the peers-level. Peers download the data from a country and forward in turn to another one; they do not reciprocate fairly with their provider peers. This corroborates our previous observation that fairness was not achieved in the P2P-TV systems (Section III).

Geographic location of peers has a considerable effect on this observation. In fact, the video streams follow a directed path among peers and it is not possible to reciprocate with data to the provider peers. Our French nodes are not able to reciprocate with data to the Asian peers because they are situated above in the play-out point of the video. Thus, they are late in the video playback and do not have any data of interest to transmit in return to Asian peers (ahead in the playback time). They can only transmit to other European peers that are in the same playback time of the video. For our Japanese nodes, they are situated in similar play-out points as the other Asian peers and can reciprocate with data.

This phenomenon could directly come from the long end-toend network delay and number of network hops from Asia to Europe since we observed previously that our French nodes download the traffic at a further distance than the Japanese nodes (section IV). It is also consistent with the fact that the downloaded traffic comes from a larger distance in number of AS hops than the upload traffic.

One could also argue that the non-reciprocal exchange of traffic is due to the asymmetric Internet connection of most of the Internet users with residential connections (DSL). However, neither our controlled nodes which have high-speed symmetric access to the Internet do not reciprocate with the same amount of data to their provider peers. For instance, with SOPCast, French peers download a lot from China but do not upload in the same proportion. This behavior is similar for PPSTream and TVAnts with Hong Kong, or for TVUPlayer with Korea.

Residential asymmetric Internet access is not the reason that prevents the reciprocal exchange between peers. The multimedia flows impose temporal constraints that result from the continuous nature of the transmission. Peers cannot transmit data in return, not necessarily because they are uncooperative, but because the nature of the content and the temporal constraints make it pointless. Since P2P systems rely on the efficient collaboration between peers, this observation is a critical issue regarding the scalability of P2P-TV systems. It is therefore of a great significant to design an incentive mechanism adapted to the continuous nature of the multimedia flows. Such a mechanism must enforce peers to collaborate in the network, even if the continuous nature of the content being distributed mitigates against the transmission of data in reciprocating manner.

\section{CONCLUSION}

In this paper, we present our P2P-TV large-scale measurement experiments that have been conducted in order to characterize the overall P2P-TV network and study the effect of this traffic on the Internet.

Our results indicate that the P2P-TV traffic is exchanged arbitrary toward many distinct locations involving many ASes, and this traffic is not kept local. This is an important issue for ISPs because it wastes their network resources by overloading the links between ISPs, increasing their costs to transport the P2P-TV traffic. The broadcasted contents have an effect on the population of peers and their geographic location. The current spread of these applications in Asia explains the large presence of Chinese peers in our traces. However, there is not necessarily a relationship between the population of peers and the volume of traffic they generate. We also find out that peers did not reciprocate fairly when downloading the traffic. This is a critical issue concerning the scalability of P2P-TV. The video streams follow a directed path among peers and it is not possible for peers to collaborate and forward data in return to their provider peers.

As a perspective of our work, we are currently designing a new incentive mechanism adapted to multimedia live streaming systems. We also plan to propose a locality-aware mechanism to select closer peers and to reduce the costs for ISPs.

\section{REFERENCES}

[1] PPSTream: http://www.ppstream.com

[2] SOPCast: http://www.sopcast.com

[3] TVUPlayer: http://www.tvunetworks.com

[4] TVAnts: http://www.tvants.com

[5] Akamai: http://www.akamai.com

[6] Cachelogic: http://www.cachelogic.com, 2006.

[7] Youtube: http://www.youtube.com

[8] D. R. Choffnes and F. E. Bustamante, "Taming the torrent: A practical approach to reducing cross-ISP traffic in peer-to-peer systems," in $A C M$ SIGCOMM, 2008.

[9] http://tools.ietf.org/html/draft-zhang-ppsp-problem-statement-05

[10] X. Hei, C. Liang, J. Liang, Y. Liu, and K. W. Ross, "Insights into PPLive: A measurement study of a large-scale P2P IPTV system," in Proc. of IPTV Workshop, WWW, 2006.

[11] T. Silverston, O. Fourmaux, A. Dainotti, A. Botta, A. Pescape, G. Ventre, and K. Salamatian, "Traffic analysis of P2P IPTV communities," Elsevier Computer Networks, March 2009.

[12] E. Alessandria, M. Gallo, E. Leonardi, M. Mellia, and M. Meo, "P2PTV systems under adverse network conditions: a measurement study," in IEEE Infocom, 2009.

[13] T. Silverston and O. Fourmaux, "Measuring P2P IPTV systems," in Proc. of ACM NOSSDAV, 2007.

[14] MyP2P: http://www.myp2p.eu

[15] http://www.cpni.gov.uk/Docs/InternetProtocol.pdf

[16] IETF RFC 3912: Whois protocol specification

[17] Cymru: http://www.team-cymru.org

[18] V. Aggarwal, A. Feldmann, and C. Scheideler, "Can ISPs and P2P systems co-operate for improved performance?" ACM SIGCOMM CCR, July 2007.

[19] Maxmind GeoIP: http://www.maxmind.com 